- L'absence ou l'inefficacité d'installation de récupération et de recombinaison d'hydrogène dans les bâtiments situés au-dessus de l'enceinte de confinement des réacteurs a conduit aux explosions qui se sont produites au-dessus des réacteurs 1 et 3 . Dans le réacteur 2 , il s'est agi d'une explosion dans le tore. Notons ici que des recombineurs d'hydrogène autocatalytiques passifs (RAP) ont été installés fin 2007 sur tous les réacteurs en service en France (voir plus bas).

- L'exploitant TEPCO a t-il disposé des moyens d'intervention adaptés aux opérations de secours dans des locaux irradiants? De même, on peut $s^{\prime}$ interroger sur le délai assez long qui a été nécessaire pour acheminer et mettre en opération des camions-pompes ayant la puissance nécessaire. Certes, les dégâts subis par les voies de communication ainsi que les délicates décisions d'affectation des moyens de secours ont dû jover un rôle, mais une analyse approfondie des raisons de ces délais s'impose. Il serait également intéressant de savoir si les robots du groupement français INTRA ${ }^{16}$ auraient été capables $d^{\prime}$ intervenir efficacement dans ce cas.

- L'absence d'enceinte de confinement au-dessus des piscines de combustible peut conduire, en cas de perte de refroidissement pendant plusieurs jours, à un danger gravissime, parce qu'à l'heure actuelle rien n'est prévu pour limiter les rejets radioactifs que cela pourrait entraîner ${ }^{17}$. On voit combien il est important de limiter autant que possible le nombre d'assemblages de combustible usé entreposés dans les piscines situées dans les bâtiments des réacteurs.

\title{
2 | La situation du nucléaire en France après Fukushima
}

L'accident de Fukushima a montré qu'un événement hautement improbable, tel une perte simultanée et sur des temps longs des alimentations électriques et des sources froides conduisant à endommager gravement trois réacteurs électronucléaires d'une centrale, est néanmoins arrivé. Il faut donc réévaluer le niveau de sûreté de nos installations nucléaires sans écarter comme impossibles des événements de très faible probabilité et sans négliger la possibilité d'occurrence simultanée de plusieurs événements rares a priori indépendants.

${ }^{16}$ Groupe d'intervention robotique sur accident, créé en 1988 par EDF-CEA-COGEMA.

17 Dans les EPR, la piscine d'entreposage est située à l'intérieur de la coque « avion » offrant ainsi une plus grande robustesse vis-à-vis d'un impact important. Mais, pour autant, cette coque ne joue aucun rôle de confinement des produits radioactifs. 
Tous les incidents passés qui se sont présentés dans l'industrie nucléaire, et a fortiori les accidents graves ou majeurs, ont conduit à un réexamen sévère des conditions de sûreté et d'exploitation des installations nucléaires. À chaque fois, cet examen a entraîné des modifications appropriées et a conduit à entreprendre des recherches visant à améliorer la sûreté et la sécurité. Tirer les leçons de Fukushima va donc s'imposer à nos activités nucléaires.

\subsection{Le parc nucléaire français}

La France produit aujourd'hui $78 \%$ de son électricité par 58 réacteurs, exploités par EDF, qui se répartissent ainsi :

- 34 réacteurs de 900 MW, moyenne d'âge 29 ans ;

- 20 réacteurs de $1300 \mathrm{MW}$, moyenne d'âge 23 ans ;

- 4 réacteurs de $1450 \mathrm{MW}$, moyenne d'âge 13 ans.

(Les moyennes d'âge citées, calculées à partir de la première divergence des réacteurs, se réfèrent à décembre 2010). Un réacteur EPR de $1600 \mathrm{MW}$ est en construction à Flamanville.

Tous ces réacteurs utilisent de l'eau sous pression (REP) pour modérer les neutrons et évacuer la chaleur, alors que ceux de Fukushima utilisent de l'eau bouillante (REB). On trouvera en annexe quelques indications sur les bases physiques du fonctionnement des réacteurs et une description plus détaillée des réacteurs à eau légère ${ }^{18}, R E P$ et $R E B$. Les réacteurs à eau présentent l'avantage qu'en cas de perte de cette eau (par vidange ou par ébullition), le nombre de réactions de fission diminue : c'est une caractéristique intrinsèque des cœurs, très importante pour la sûreté. Ces réacteurs utilisent de l'uranium enrichi à $3,5 \%{ }^{19}$ et en plus, pour 20 de ces 58 réacteurs, du combustible MOX (Mixed oxide fuel, voir annexe 14).

La conception, la construction, l'exploitation et le démantèlement des installations nucléaires (réacteurs nucléaires, ateliers du cycle du combustible nucléaire) sont évidemment guidés par des impératifs de sûreté. La responsabilité de la sûreté des installations est assurée par l'exploitant, c'està-dire EDF pour les réacteurs électrogènes. Les modifications permettant d'améliorer la sûreté des installations interviennent lors :

- du retour d'expérience consécutif aux incidents et accidents ;

- des visites décennales imposées à toutes les installations nucléaires.

\footnotetext{
${ }^{18}$ |l existe également des réacteurs à eau " lourde ", où une fraction des molécules d'eau est composée d'oxygène et de deutérium, isotope deux fois plus lourd de l'hydrogène.

${ }^{19}$ Voir l'annexe 2 : glossaire nucléaire.
} 
Les incidents qui émaillent la vie de toute industrie, telle l'inondation de la centrale du Blayais en 1999, et surtout les accidents graves comme celui de Three Mile Island (TMI) en 1979, ou majeurs comme celui de Tchernobyl en 1986, donnent donc lieu à une analyse approfondie et conduisent à des améliorations significatives de la sûreté, au plan technique mais aussi au plan organisationnel et humain. C'est en France l'une des missions de la R\&D et de I'ingénierie d'EDF, I'IRSN y travaillant aussi naturellement. II est évident que I'accident de Fukushima conduira d'abord à une revue des risques du même type puis à la mise en place si nécessaire des mesures correctrices.

L'autorisation de construction d'un réacteur n'impose pas de limite de durée a priori mais exige que l'exploitant se soumette à un examen de sûreté approfondi tous les dix ans ${ }^{20}$. La remise en fonction du réacteur est soumise à la décision de l'Autorité de sûreté nucléaire (ASN). Les réacteurs les plus anciens sont donc soumis actuellement à leur troisième visite décennale, commencée avec les réacteurs $n^{\circ} 1$ de Tricastin et $n^{\circ} 1$ de Fessenheim. L'ASN a approuvé en novembre 2010 la poursuite $d^{\prime}$ exploitation du réacteur $n^{\circ} 1$ de Tricastin pour une durée de 10 années supplémentaires après 30 ans de fonctionnement. Elle prendra position en 2011 sur l'aptitude du réacteur $n^{\circ} 1$ de Fessenheim à être exploité jusqu'à sa prochaine visite décennale. Le réacteur $n^{\circ} 2$ de Fessenheim est à son tour en arrêt décennal.

La surveillance continue des réacteurs, les modifications apportées pour tenir compte du retour d'expérience et des progrès des recherches sur la sécurité, la visite décennale imposée avec redémarrage subordonné à l'ASN, ont pour effet de réduire sensiblement les risques potentiels dus au vieillissement des installations nucléaires.

L'accident de Fukushima n'est donc pas un motif d'arrêt de nos réacteurs les plus anciens ${ }^{21}$. En revanche, il implique l'inspection approfondie de toutes nos installations nucléaires (jeunes ou vieilles) en tant que systèmes technologiques complexes, avec une attention particulière à porter sur les systèmes de contrôle et de sauvegarde, les équipements annexes et les piscines d'entreposage de combustibles usés. Il conviendra de réexaminer toutes les piscines d'entreposage, celles qui jouxtent les réacteurs comme celles où sont entreposés les déchets en attente de vitrification et stockage.

${ }^{20}$ Nous nous sommes demandés pourquoi ce délai de dix ans, pourquoi pas cinq par exemple? En fait la mise en place d'un examen décennal demande plusieurs années d'études préparatoires et les travaux correspondants prennent aussi du temps! De plus, la sûreté fait l'objet d'une surveillance permanente.

${ }^{21}$ Le NRC (Nuclear Regulatory Commission des États-Unis) examine depuis 2002 les demandes d'extension de 40 à 60 ans des licences d'exploitation des réacteurs nucléaires. Cette extension a déjà été accordée à environ la moitié du parc des États-Unis qui est constitué de plus de 100 réacteurs. Les possibilités d'extension à 80 ans y sont déjà à l'étude. 
Par ailleurs il faut bien entendu également se prémunir contre la possibilité $d^{\prime}$ 'une sécheresse sévère ne permettant plus $d^{\prime}$ 'assurer convenablement le refroidissement. Mais le danger n'est pas de même nature. On peut la voir venir et arrêter en cas de nécessité les réacteurs; ce serait une pénurie d'électricité mais rien de comparable à un tsunami ou à une inondation brutale.

Notons que sur chaque site des centrales en France, il y a des systèmes de réserve d'eau de refroidissement du réacteur et de l'ensemble de l'usine, mis en place dès la conception. Ils ont été parfois améliorés lorsque cela s'est révélé nécessaire compte tenu du retour d'expérience. L'accident de Fukushima doit être à son tour pris en compte pour d'éventuelles améliorations.

\subsection{L'organisation de notre dispositif de sûreté}

\subsubsection{La situation réglementaire}

La loi du 13 juin 2006, relative à la transparence et à la sécurité en matière nucléaire, a créé l'ASN, autorité administrative indépendante du gouvernement, chargée de contrôler toutes les activités nucléaires civiles. Celle-ci assure, au nom de l'État, le contrôle de la sûreté nucléaire et de la radioprotection en France pour protéger les travailleurs, les patients, le public et l'environnement des risques liés à l'utilisation du nucléaire ${ }^{22}$ et des rayonnements ionisants. L'ASN est placée sous le contrôle de cinq commissaires irrévocables, nommés pour six ans par le président de la République et les présidents des deux assemblées parlementaires. La même loi du 13 juin 2006 a institué des Commissions locales $\mathrm{d}^{\prime}$ information $(\mathrm{CLI})^{23}$ auprès de chaque installation nucléaire.

Autant que nous ayons pu en juger, l'ASN est une autorité qui démontre son indépendance en exerçant un contrôle effectif rigoureux sur les installations nucléaires civiles. Par exemple, c'est l'injonction de l'ASN qui a contraint I'opérateur EDF à arrêter le réacteur $n^{\circ} 3$ de Bugey jusqu'au remplacement des générateurs de vapeur à la suite de la découverte d'une corrosion importante sur l'un d'entre eux. Le réacteur a été arrêté pendant vingt mois.

Notons également que, depuis 2001, un délégué à la sûreté nucléaire et à la radioprotection pour les activités et installations intéressant la défense (DSND) est placé auprès du ministre de la Défense et du ministre chargé de l'Industrie.

${ }^{22}$ La sécurité nucléaire comprend la sûreté nucléaire, la radioprotection, la prévention et la lutte contre les actes de malveillance, ainsi que les actions de sécurité civile en cas d'accident.

${ }^{23}$ Voir le site de l'Association nationale des comités et commissions locales d'information : www.anccli.fr 
L'ASN et le DSND s'appuient sur le support technique que lui fournit l'Institut de radioprotection et de sûreté nucléaire (IRSN).

\subsubsection{La recherche en matière de sûrreté nucléaire}

C'est par une loi du 9 mai 2001 qu'a été créé I'Institut de radioprotection et de sûreté nucléaire (IRSN), qui comporte actuellement environ 1000 spécialistes, chercheurs, ingénieurs, techniciens, médecins, compétents dans l'ensemble des domaines concernés par les activités nucléaires et la radioprotection. Les recherches en matière de sûreté nucléaire que mène cet Institut pour nourrir ses expertises au profit des pouvoirs publics sont conduites à l'intérieur des laboratoires de I'IRSN implantés sur onze sites en France et comportent souvent des partenariats avec les industriels (EDF et Areva), le CEA ${ }^{24}$, le CNRS et de nombreux laboratoires internationaux ${ }^{25}$. L'IRSN consacre $90 \mathrm{M}$ /an à ses programmes de recherche en sûreté, réalisés en grande partie dans ses laboratoires et ceux de ses partenaires. L'IRSN conduit également des recherches en radioprotection de l'homme et de l'environnement, dont les résultats ont été mis à profit à l'occasion de Fukushima. À titre d'exemple, voir en annexe 9 le texte concernant l'apport des recherches de I'IRSN sur les accidents avec fusion du cœur.

\subsubsection{Les programmes français de recherche en matière de sûreté nucléaire}

L'objectif majeur qui guide la conception, le dimensionnement et l'exploitation des installations nucléaires (réacteurs nucléaires, ateliers du cycle du combustible nucléaire) est $d^{\prime}$ 'assurer la sûreté de ces installations en prenant en compte, dès la conception, des dispositions pour parer à certains accidents potentiels. Pour les réacteurs, l'accident le plus redouté est la fusion du cœur car elle peut conduire à des rejets radioactifs conséquents dans l'environnement.

Les recherches pour comprendre l'enchaînement des phénomènes qui conduisent à des rejets radioactifs accidentels et leurs conséquences sur l'environnement sont indispensables à la mise en œuvre de l'énergie nucléaire de fission. En France, ce sont I'IRSN et le CEA qui les conduisent en relation

${ }^{24}$ Commissariat à l'énergie atomique et aux énergies alternatives.

${ }^{25}$ De nombreuses références aux programmes de l'IRSN sont sur le site irsn.fr. Le document de M. Schwarz "Recherche à I'IRSN sur les accidents de fusion de cœur », annexé au rapport, fait un point en avril 2011. 
avec les exploitants concernés (EDF pour les réacteurs, Areva pour les installations du cycle du combustible, CEA pour les réacteurs de recherche et autres installations nucléaires de base). L'IRSN et le CEA participent à de nombreux programmes européens ou internationaux et en pilotent certains. La recherche effectuée ou pilotée par l'IRSN est essentielle au développement de ses capacités d'expertise indépendante. Les exploitants ont eux-mêmes leurs propres équipes de recherche et développement.

Ces recherches ont souvent été intensifiées après des incidents ou accidents intervenus sur des réacteurs ou des installations du cycle. Chaque accident révèle des situations nouvelles et conduit à des progrès en matière de sûreté. Par exemple, suite aux accidents de TMI-2 (1979) et Tchernobyl (1986), les programmes de recherche et le retour d'expérience ont entraîné des modifications importantes des composants assurant la sûreté lou elles sont en cours de réalisation) sur les réacteurs actuels de $2^{\mathrm{e}}$ génération ainsi que le développement de systèmes destinés à en limiter les conséquences (recombineurs d'hydrogène, éventage et filtration de l'enceinte de confinement). De nouvelles procédures de conduite des réacteurs ont également été mises en place. L'ensemble des enseignements et des recherches sur les accidents a contribué à la conception des réacteurs de $3^{e}$ génération, tel I'EPR.

Les programmes de recherches dans le domaine de la sûreté relatifs aux réacteurs nucléaires de $2^{e}$ et $3^{e}$ générations concernent deux types $d^{\prime}$ accident :

- les accidents de dimensionnement, ceux dont les conséquences sont prises en compte dès la conception des réacteurs. L'enjeu de l'étude de ces accidents est de parer à ce qu'ils ne dégénèrent pas en accidents graves. Ils comportent deux grandes catégories : les accidents de perte de réfrigérant primaire (problématique du refroidissement en cas de rupture de tuyauterie primaire) et les accidents de réactivité laugmentation instantanée de puissance au sein du réacteur par éjection d'une barre de contrôle de la réactivité, entraînant une montée en température des combustibles et un chargement mécanique de la gaine très rapides) ;

- les accidents graves ou accidents hors dimensionnement (c'est-à-dire non pris en compte lors de la conception), pour lesquels l'enjeu est de maîtriser leurs impacts. Il s'agit d'évaluer le risque de perte de confinement consécutif à la fusion partielle ou totale du cœur du réacteur, et de prévoir à l'avance les dispositifs de prévention afin de limiter les conséquences (mitigation). De tels accidents avec fusion de cœur n'ont pas été pris en compte lors de la conception de la $2^{\mathbf{e}}$ génération de réacteurs, de sorte que les programmes d'étude ont pour objet la réduction de ces risques et la limitation de leurs conséquences. 
À la suite de la demande des autorités de sûreté en 1993, les accidents graves doivent être pris en compte à la conception pour tout nouveau projet de réacteur. En particulier, les dispositions permettant de circonscrire les conséquences de ces accidents à l'intérieur de l'enceinte de confinement du réacteur sans porter atteinte à son intégrité, sont prises en compte pour la conception des réacteurs de $3^{\mathrm{e}}$ génération comme I'EPR.

La recherche dans le domaine de la sûreté se heurte à l'extrême complexité des phénomènes mis en jeu. Les objectifs scientifiques sont de parvenir à comprendre au mieux les processus physiques et chimiques qui conduisent à la rupture des barrières de confinement Igaine des crayons de combustible, circuit primaire et enceinte de confinement) et à la caractérisation des rejets consécutifs (radionucléides émis, quantités, dispersion dans l'environnement, mesures de terrain). Il faut être capable de développer les modèles et des outils de simulation. Ils doivent notamment permettre de prévoir le déroulement d'un accident dans sa globalité et d'évaluer la pertinence des moyens mis en œuvre pour en limiter les conséquences.

Les programmes du CEA en soutien à la sûreté de l'électronucléaire sont principalement financés par les exploitants, par la subvention de l'État et, pour certains, par l'IRSN.

Il est indispensable de pouvoir effectuer ces recherches d'intérêt public, même au-delà de celles qui sont réalisées ou soutenues par les industriels.

Les recherches expérimentales dans le domaine de la sûreté demandent, notamment pour celles concernant le combustible, des moyens importants, au sein $d$ 'installations permettant de mettre en œuvre de la matière très radioactive, ce qui est seulement possible, en France, dans les installations du CEA. Ces moyens expérimentaux destinés aux recherches sur la sûreté doivent être pour certains rénovés et de nouvelles installations sont en construction.

Ce que l'on sait du comportement d'un réacteur non refroidi permet de comprendre ce qui s'est passé à Fukushima. L'accident de Fukushima, tel qu'il peut, ou pourra, être analysé, montre que certains événements appellent un approfondissement des connaissances voire de nouvelles recherches. Le CEA, I'IRSN et les industriels examinent d'ores et déjà comment compléter ou réorienter certaines de leurs recherches et établir les priorités et les financements.

Quelles que soient les circonstances conduisant à des rejets de matière radioactive, il est important de pouvoir rapidement caractériser la contamination et son étendue. II y a là un domaine de recherche qui doit être abordé par une large communauté scientifique car il relève de problèmes d'environnement. Il faut améliorer la modélisation opérationnelle des éléments 
radioactifs contenus dans le combustible, aussi bien que l'étude de leur chimie et de leur transport dans l'environnement.

La recherche en matière de sûreté nucléaire est une priorité qui doit être inscrite dans des programmes clairement affichés et ces programmes doivent être portés à la connaissance du public. En particulier, la recherche publique en matière de sûreté doit être fortement réactivée et développée audelà de celle qui est réalisée ou soutenue par les industriels. Elle doit concerner tant la physicochimie des accidents que la gestion de crise d'un accident grave et les moyens de mitigation de ce dernier. La communauté scientifique doit y être associée au-delà des organismes dédiés comme le CEA ou I'IRSN.

\section{Questions et recherches concernant l'hydrogène}

Les explosions d'hydrogène révèlent un risque réel en cas de fusion du cœur, qui paraît avoir été insuffisamment maîtrisé dans le cas de la centrale de Fukushima. Ce risque est bien identifié dans de nombreuses études en France et dans le monde. Des dispositifs de recombinaison passifs de l'hydrogène ont été placés sur toutes les centrales françaises pour consommer ce gaz au fur et à mesure de son relâchement et éviter son accumulation en cas de production accidentelle. De manière générale, la recombinaison effectuée par catalyse est lente par rapport au taux de production d'hydrogène dans le cas d'une fusion de cœur. Il faudrait vérifier que les dispositions prises permettent cependant de limiter la quantité d'hydrogène présente temporairement dans l'enceinte de confinement.

Il serait aussi utile d'examiner le comportement des dispositifs d'éventagefiltration qui sont installés sur les réacteurs existants pour réaliser une dépressurisation et limiter la pression dans l'enceinte de confinement. Bien que l'objectif premier ne soit pas d'évacuer l'hydrogène, une inflammation de ce gaz pourrait survenir en cours d'éventage. Les dispositions prises pour éviter ce phénomène consistent à utiliser la forte teneur en vapeur d'eau pour inerter le mélange et à placer des dispositifs de préchauffage pour éviter la condensation de la vapeur dans les tuyauteries et maintenir l'inertage de l'écoulement. Ces dispositions devraient faire l'objet d'un réexamen à la lumière de l'accident de Fukushima. Plus généralement, il est important de poursuivre les actions de recherche en cours sur la réduction des risques associés à l'hydrogène dans les enceintes de confinement. 